\title{
Opiate addiction in adult offspring through possible imprinting after obstetric treatment
}

\author{
Bertil Jacobson, Karin Nyberg, Leif Grönbladh, Gunnar Eklund, Marc Bygdeman, Ulf Rydberg
}

\section{Abstract}

Objective-To test the hypothesis that opiate addiction in adults might stem partly from an imprinting process during birth when certain drugs are given to the mother.

Design-Retrospective study by logistic regression of opiate addicts with siblings as controls.

Setting-Stockholm, Sweden.

Subjects -200 Opiate addicts born in Stockholm during 1945-66, comprising 41 identified during interviews of probands for an earlier study; 75 patients whose death from opiate addiction had been confirmed during 1978-88; and 84 accepted for the methadone programme. 262 Siblings (controls) born in Stockholm during the same period, 24 of whom were excluded for drug addiction or being brought up outside the family. Birth records were unavailable for eight, leaving 230 siblings and 139 corresponding probands.

Main outcome measures-Administration of opiates, barbiturates, and nitrous oxide (for $>1 \mathrm{~h}$ ) to mothers of all subjects during labour within 10 hours before birth as a risk factor for adult opiate addiction.

Results-In subjects who had subsequently become addicts a significant proportion of mothers had received opiates or barbiturates, or both, compared with unmatched siblings $\left(25 \% v 16 \%, \chi^{2}=5 \cdot 83, \mathrm{df}=1\right.$, $\mathbf{p}=\mathbf{0 . 0 2}$ ), and these mothers had received nitrous oxide for longer and more often. After controlling for hospital of birth, order of birth, duration of labour, presentation other than vertex, surgical intervention, asphyxia, meconium stained amniotic fluid, and birth weight the relative risk for offspring subsequently becoming an adult opiate addict increased with the number of administrations of any of the three drugs. When the addicts were matched with their own siblings the estimated relative risk was $4.7(95 \%$ confidence interval 1.8 to 12.4 , $p$ for trend $=0.002$ ) for three administrations compared with when no drug was given.

Conclusions-The results are compatible with the imprinting hypothesis. Therefore, for obstetric pain relief methods are preferable that do not permit substantial passage of drugs through the placenta.

\section{Introduction}

Imprinting is an ethologically well documented process, ${ }^{\prime 2}$ which has been shown in insects, fish, reptiles, birds, and mammals. Imprinting has also been considered to be important in humans, ${ }^{3+}$ but there is little statistical evidence for the existence of imprinting in the ethological sense-that is, a specific memory engraved during a short sensitive period, of the order of hours, leading to behavioural effects in adult life. It has been suggested, however, that self destructive behaviour, such as suicide and amphetamine addiction, might stem partly from an imprinting process at birth. ${ }^{56}$ The purpose of the present study was to explore further the possibility that imprinting might be a mechanism contributing to opiate addiction.

In accordance with the imprinting hypothesis depressant and sedative drugs, such as opiates and barbiturates, would be expected to constitute risk factors for opiate addiction. An indication that this might be the case was obtained in a pilot study, ${ }^{5}$ but its design did not take into account genetic and socioeconomic confounders; these are considered in this study comparing opiate addicts and matched biological siblings.

As a matter of statistical method it is essential that the expected association between administered opiates and barbiturates was already suggested before performing the present investigation (the evidence for this was presented to the ethics committee of the Karolinska Institute). The number of subjects and the method of analysis (logistic regression of opiate addicts matched with siblings) were also determined in advance.

\section{Subjects and methods} ADDICTS AND CONTROLS

The birth records for 200 opiate addicts born in Stockholm during 1945-66 were gathered from three sources: (a) 41 opiate addicts identified when interviewing probands at the Stockholm County Custody for a study of amphetamine addiction ${ }^{6}$ (of the total of 260 addicts of then unknown drug preference asked to participate, $11(4 \cdot 2 \%)$ refused and birth records could not be found for one opiate addict $(2 \cdot 4 \%)) ;(b)$ all 75 unambiguous cases of death from opiate addiction for which necropsies had been performed at the State Institute of Forensic Medicine in Stockholm during 1978-88, and in which, according to medical records at the county custody, the subjects had been categorised as opiate users (among a total of 80 subjects, birth records could not be retrieved for five $(6 \cdot 3 \%))$; and $(c)$ 84 opiate addicts accepted for the methadone programme at the Ulleråker Hospital (the first subjects responding were included to obtain the required total of 200 subjects; of a total of 120 , another $31(26 \%)$ were originally considered for participation but not included, and of 89 addicts the birth records were missing for five $(5 \cdot 6 \%)$.

Two hundred and sixty two siblings were born in Stockholm during 1945-66, of whom, 24 were also drug addicts or had been brought up outside the family, and they were consequently excluded from the study. For eight of the remaining siblings, birth records could not be retrieved, yielding 230 siblings for comparison with 139 probands who had siblings. The proportions of males among the addicts and their siblings were $74 \%$ and $48 \%$, respectively.

\section{MISSING DATA}

Data for administration of nitrous oxide were missing in $55(28 \%)$ of the subjects and $56(24 \%)$ of the siblings. 
The origin of the missing data had been shown in a previous study to be related to certain midwives ${ }^{6}$ and was not likely to be owing to systematic errors causing bias. For data on opiates and barbiturates no similar analysis can be done as no entries for these drugs existed in the preprinted forms but were recorded in longhand. Yet, these drugs were likely to have been more accurately recorded than nitrous oxide as, contrary to nitrous oxide, they were given only by the obstetrician's prescription.

\section{BLIND EVALUATION}

Copies of the birth records of the addicts and siblings were coded and ordered by hospital and year of birth. The data were extracted by a hired experienced midwife (GL), who was unaware of the specific hypothesis being tested. (In Sweden midwives, and not doctors, assist most births and are responsible for completing all birth records.)

\section{DRUG DOSAGE}

Opiates were given in doses of $0 \cdot 01-0.02 \mathrm{~g}$ morphine or $0 \cdot 05-0 \cdot 1 \mathrm{~g}$ pethidine hydrochloride, and barbiturates 0.05-2 g phenobarbitone (and in a few instances other types of barbiturates). When coding the variables in the following strata, administrations were considered only when given within 10 hours before delivery: 0 , no dose; 1, a single dose; or 2, multiple doses. Nitrous oxide was stratified in four levels relative to the dose for $\leqslant 0.25 \mathrm{~h}: 1,>0.25 \mathrm{~h}$ to $\leqslant 1 \mathrm{~h} ; 2,>1 \mathrm{~h}$ to $<2.5 \mathrm{~h} ; 3$, $\geqslant 2.5 \mathrm{~h}$ to $<4.5 \mathrm{~h}$; and $4, \geqslant 4.5 \mathrm{~h}$, which corresponded to those used in the study of amphetamine addiction, and yielded an approximate geometric series of mean exposures.

A combined drug score was defined as the total number of administrations within 10 hours before birth of opiates or barbiturates or nitrous oxide for $\geqslant 1 \mathrm{~h}$, or any such combination. (If nitrous oxide was given for $<1 \mathrm{~h}$ it was not included in the score because of the apparent minor risk associated with brief exposures, as estimated by logistic regression analysis in this study.) When calculating the score a missing nitrous oxide value was interpreted as if no gas had been administered. The score varied between 0 and 3 according to the levels indicated above.

\section{STATISTICAL METHODS}

The LOGREG procedure in the EPILOG epidemiological package was used for logistic regression ${ }^{7}$ of addicts with a variable number of siblings as controls. Confounders were excluded by stepwise regression. Determined regression coefficients permitted estimation of odds ratios (relative risk factors) for various factors, taking confounders into account.

\section{Results}

In subjects who had subsequently become addicts a significant proportion of mothers had received opiates or barbiturates, or both, when giving birth when compared with unmatched siblings $\left(25 \% v 16 \%, \chi^{2}=\right.$ $5 \cdot 83 ; \mathrm{df}=1, \mathrm{p}=0.02$ ). Table I shows the time between administration of opiates and barbiturates and delivery. If the drugs had been administered more than 10 hours before delivery no significant difference between the groups was observed. In general, mothers had received nitrous oxide for longer periods more often during delivery of those who subsequently became addicts than during that of their siblings (table II).

Analysis of the separate administration of opiates or barbiturates to mothers by matched regression (139 addicts and 230 siblings) yielded, for single doses, relative risks of $1.6(95 \%$ confidence interval 0.75 to 3.6 , $\mathrm{p}=0.2)$ and $1.7(0.97$ to $3.00, \mathrm{p}=0.06)$, respectively, and by unmatched regression ( 200 addicts and 230
TABLE I - Administration of opiates or barbiturates, or both, to mothers of subsequent addicts and unmatched siblings, according to time of administration. Figures are numbers (percentages) ${ }^{\star}$ unless otherwise stated

\begin{tabular}{|c|c|c|c|}
\hline \multirow[b]{2}{*}{$\begin{array}{l}\text { Time before } \\
\text { birth (h) }\end{array}$} & \multicolumn{3}{|c|}{ Opiates + barbiturates $f$} \\
\hline & $\begin{array}{l}\text { Addicts } \\
(\mathrm{n}=200)\end{array}$ & $\begin{array}{l}\text { Siblings } \\
(\mathrm{n}=230)\end{array}$ & $\begin{array}{c}\text { Ratio of } \\
\text { percentages }\end{array}$ \\
\hline$\leqslant 0 \cdot 5$ & $1+0=1$ & $1+1=2(0.9)$ & $0.6 \ddagger$ \\
\hline$>0 \cdot 5-\leqslant 1.5$ & $1+5=6$ & $2+0=2 \quad(0 \cdot 9)$ & $3 \cdot 5$ \\
\hline$>1 \cdot 5-\leqslant 4 \cdot 5$ & $11+12=23(11 \cdot 5)$ & $6+10=16(7 \cdot 0)$ & $1 \cdot 7$ \\
\hline$>4 \cdot 5-\leqslant 10$ & $7+6=13 \quad(6 \cdot 5)$ & $2+4=6 \quad(2 \cdot 6)$ & $2 \cdot 5$ \\
\hline$>10$ & $9+9=18 \quad(9 \cdot 0)$ & $5+11=16(7 \cdot 0)$ & $1 \cdot 3$ \\
\hline No administration & $150(75 \cdot 0)$ & $194(84 \cdot 3)$ & 0.89 \\
\hline
\end{tabular}

* Sum of percentages exceeds 100 as some mothers received both drugs. †Only dose closest to delivery was considered.

$\ddagger$ Therapeutic pharmacological effect is unlikely because of short term drug delivery interval.

TABLE II-Numbers (percentages) of addicts and unmatched siblings according to duration of nitrous oxide treatment of mothers during labour

\begin{tabular}{lccc}
\hline $\begin{array}{l}\text { Duration of } \\
\text { nitrous oxide } \\
\text { treatment }(\mathbf{h})\end{array}$ & $\begin{array}{c}\text { Addicts } \\
(\mathbf{n}=145)\end{array}$ & $\begin{array}{c}\text { Siblings } \\
(\mathbf{n}=174)\end{array}$ & $\begin{array}{c}\text { Ratio of } \\
\text { percentages }\end{array}$ \\
\hline$\leqslant 0 \cdot 25$ & $22(15 \cdot 2)$ & $44(25 \cdot 3)$ & $0 \cdot 6$ \\
$>0 \cdot 25-\leqslant 1 \cdot 0$ & $30(20 \cdot 7)$ & $46(26 \cdot 4)$ & $0 \cdot 8$ \\
$>1 \cdot 0-<2 \cdot 5$ & $32(22 \cdot 1)$ & $32(18 \cdot 4)$ & $1 \cdot 2$ \\
$\geqslant 2 \cdot 5-<4 \cdot 5$ & $30(20 \cdot 7)$ & $30(17 \cdot 2)$ & $1 \cdot 2$ \\
$\geqslant 4 \cdot 5$ & $31(21 \cdot 4)$ & $22(12 \cdot 6)$ & $1 \cdot 7$ \\
\hline
\end{tabular}

siblings) $1 \cdot 8(0 \cdot 94$ to $3 \cdot 5, \mathrm{p}=0 \cdot 08)$ and $1 \cdot 6(0 \cdot 97$ to $2 \cdot 6$, $\mathrm{p}=0 \cdot 07)$, respectively. Considering only addicts and controls for whom data on nitrous oxide were available gave in a matched regression analysis ( 99 addicts and 174 siblings), for a duration of treatment of $>1 \mathrm{~h}$ to $<2.5 \mathrm{~h}$, an estimated relative risk of $1.6(0.95$ to 2.6 , $\mathrm{p}=0.08)$ and in an unmatched regression analysis $(145$ addicts and 174 siblings) a risk of $1 \cdot 7(1 \cdot 2$ to $2 \cdot 3$, $\mathrm{p}=0.003)$. Similarly when analysing the administration of chloroform, only addicts for whom data had been recorded were included. The effect of chloroform was found insignificant; for matched data, regression analysis ( 108 addicts and 184 siblings) for a dose of $5 \mathrm{~g}$, showed an estimated risk of $1.02(p=0.94)$.

For multivariate analysis the combined drug score was tested by conditional logistic regression (probands were matched with their own siblings) in competition with the following eight potential confounders: hospital of birth, order of birth, duration of labour, presentation other than vertex, surgical intervention (forceps or vacuum extraction or caesarean section), asphyxia, meconium stained amniotic fluid, and birth weight. During the stepwise procedures, only the combined drug score was significant ( $p=0.002$, test for trend).

The figure shows the estimated and observed relative risks for one, two, and three drug administrations

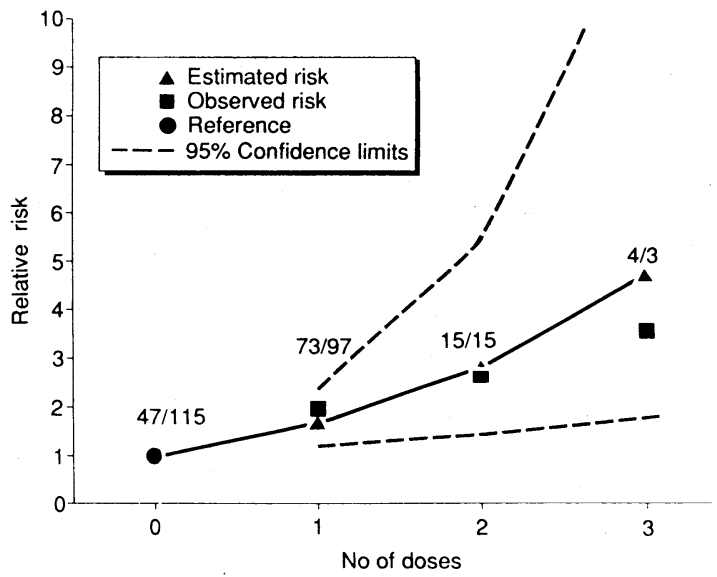

Relative risk factors (odds ratios) for one to three administrations of opiates and/or barbiturates and/or nitrous oxide for $\geqslant 1 \mathrm{~h}$ within 10 hours before birth. Figures are numbers of opiate addicts/siblings 
compared with no drugs. For a single administration the relative risk was $1.7(1 \cdot 2$ to $2 \cdot 3)$ times higher than if none of the three drugs had been given. For the highest stratum with three administrations the relative risk was $4 \cdot 7(1 \cdot 8$ to $12 \cdot 4)$.

To exclude sex as a confounder an unmatched test was performed of the combined drug score for male addicts and male siblings; the relative risk factor for a single administration was $1.7(1 \cdot 2$ to $2 \cdot 5, \mathrm{p}=0.003)$ compared with $1.6(0.95$ to $2.6, p=0.08)$ for female subjects.

The time of administration of opiates or barbiturates, or both, before delivery (stratified in three levels relative to that for $>10 \mathrm{~h}$ or "no drugs given": $1=\leqslant 10 \mathrm{~h}$ to $>4.5 \mathrm{~h} ; 2=\leqslant 4.5 \mathrm{~h}$ to $>1.5 \mathrm{~h}$; and $3=\leqslant 1.5 \mathrm{~h}$ ) gave in matched regression an estimated relative risk of $2 \cdot 5(1 \cdot 1$ to $6 \cdot 2, p=0 \cdot 04$, test for trend $)$ when any of the two drugs had been given within 1.5 hours before birth compared with $>10$ hours, including "no drugs given."

\section{Discussion}

The quality of the birth records varied, and in some instances data were missing. This cannot explain, however, the observed lower rate of administration of drugs to siblings; it is difficult to imagine a mechanism by which the record keeping for the siblings would have been less accurate than for the addicts. If a notation about drug administration was made, it very probably meant that the drug was actually given. Furthermore, any inaccuracy in records would have tended to attenuate the observed differences between the groups not enhance them; consequently, we regard the validity of the data as being sufficient.

Interpreting missing data on administration of nitrous oxide as equivalent to the drug not having been administered probably reduces the contribution of this drug when estimating the relative risk. There seems to be no reason to assume any systematic error because of this assumption as the percentage frequency of missing data for both addicts and siblings was similar $(28 \%$ and $24 \%$, respectively).

In two earlier studies ${ }^{5}$ administration of opiates and barbiturates was considered only if these were to be given within 10 hours before birth. The limit was chosen on theoretical grounds that imprinting would occur close to birth. The validity of the limit, which has been retained in the present study, is supported by the observation in table I that no significant difference was observed for larger intervals between administration and delivery.

The observed distribution of the interval between administration of opiates and barbiturates and delivery showed a maximum of cases in the $>1.5-\leqslant 4.5 \mathrm{~h}$ interval (table I). This is in agreement with kinetic studies on pethidine, in which appreciable concentrations of pethidine were found in infants' plasma when a dose of $100 \mathrm{mg}$ had been administered at $0 \cdot 5-2 \cdot 5 \mathrm{~h}$ before delivery. ${ }^{8}$ The distribution suggests that the effect associated with addiction in offspring does not depend on metabolites of the drug but on the drug itself.

Although asphyxia at birth was found to be unimportant, we cannot exclude that minimal brain damage was caused by hypoxia during administration of $100 \%$ nitrous oxide well before birth. Such an injury might contribute to an emotional instability leading to a greater susceptibility to drug addiction.

The results support the hypothesis formulated before the study that opiates and barbiturates are risk factors for opiate addiction in offspring. Nitrous oxide, the only drug associated with amphetamine addiction in offspring, according to the previous study, ${ }^{6}$ was not suspected to be a risk factor for opiate addiction. But the results indicate that, indeed, nitrous oxide also contributes to this risk. The distribution shown in table II, which closely resembles the corresponding distribution in the study of amphetamine addiction, suggests a dose-response effect. In retrospect there are similarities in the actions of opiates and nitrous oxide that make the association reasonable. Nitrous oxide in mice elicits its effects through two mechanisms." One of these is mediated by catecholamines, particularly dopamine, and is similar to that found for amphetamines. ${ }^{10}$ This might be the mechanism behind the elating sensation, which, when imprinted, could be one of the explanations underlying preference for amphetamines. The other is mediated by opioid mechanisms, and is consequently similar to those mechanisms for opiates. Hence it is conceivable that nitrous oxide may be associated with both types of addiction.

To assume that infants may be imprinted is a simple analogy to what is known in great detail for animals. ${ }^{23}$ In humans imprinting should be understood as leading to a gratifying emotional state when re-experiencing the sensations felt during the sensitive imprinting period. This might be one explanation why certain people are more easily trapped into dependence when testing drugs under peer pressure. Thus we do not believe that addiction itself is imprinted but a propensity to use or misuse opiates. When seeking for the imprinting stimulus mammals and birds show an apparent anxiety; in the presence of this stimulus their wellbeing seems to be increased. Relief of anxiety through re-experiencing the imprinted stimulus might be one of the rewards for which addicts strive.

We could not formulate an alternative to the imprinting hypothesis that explains equally well the findings in this study and some earlier observations.

(1) The demonstrated increased risk for administration of opiates or barbiturates close to delivery is to be expected if imprinting is the underlying mechanism. The sensitive hours for imprinting in humans are probably close to birth, as catecholamine concentrations are then maximal" and such hormones facilitate imprinting during this period..$^{12} 13$

(2) Our results do not confirm that chloroform is a risk factor for drug addiction, as suspected in the pilot study. ${ }^{5}$ In terms of imprinting this makes sense as chloroform, contrary to the other drugs considered here, gives few subjective sensations apart from reduced consciousness.

(3) The three tested drugs have different chemical structures, yet they contribute about equally to the risk. This is understandable if the effect is mediated through a mental experience leading to an imprinted memory rather than through a toxic effect on brain tissue.

(4) Drug addiction shows male dominance, which is to be expected as male children have higher concentrations of circulating testosterone at birth, and this hormone, among other substances, facilitates imprinting.

(5) Imprinting has been described as an irreversible memory process, and it causes specific neuroanatomical changes in the brain of animals. ${ }^{2}$ The remarkable stability of an imprinted memory might be one of the explanations for the well known difficulties of treating addiction and the difficulty of avoiding relapses.

(6) The first generations exposed to heavy analgesia and sedation at birth were born in the mid-1940s and later. A pronounced increase in drug misuse occurred during the 1960s, and the imprinting hypothesis suggests that there might be a causal connection.

Imprinting is certainly only one possible causative mechanism for drug addiction; it does not exclude many others. It should be emphasised, however, that the other perinatal factors tested in this study cannot explain the results, as these confounders were 
controlled for in the multivariate analysis. Furthermore, the suggested risk factors occurring later in life, such as psychological mechanisms and drug availability and exposition, cannot explain the demonstrated associations between administration of drugs during the perinatal period and subsequent addiction in offspring. Also, genetic and socioeconomic factors were largely controlled for by matching addicts with their own siblings.

In conclusion, effective pain relief is sometimes an important factor for a successful outcome of delivery, as well as for the ability of the mother to accept and care for the child in the future. At present, when considering the choice of analgesic method immediate risks and benefits are mainly taken into account. When depressant or sedative drugs are used their possible long term effects due to imprinting also seem to be important. From this point of view, analgesic methods not associated with passage of substantial amounts of drugs across the placenta are preferable. Our results are compatible with the imprinting hypothesis, and we have not found reason to reject it. This study and the previous study on amphetamine addiction need to be repeated by other investigators. We consider it essential that siblings are then used as controls as we have some evidence of family related risk factors, which we shall publish elsewhere; to control for such factors it is mandatory to match probands with siblings.

This study was approved by the ethics committees of the Karolinska Institute and Uppsala University and was supported by the Expressen Prenatal Research Foundation. We thank Professor Lars Gunne, Ulleråker University Hospital, Uppsala, and Professor Lars Terenius, Karolinska Institute, for their views on the effects of nitrous oxide and their helpful suggestions.

1 Lorenz K. Der Kumpan in der Umwelt des Vogels. Fournal of (Omuthology $1935 ; 83: 137-213,289-413$

2 Horn $G$. Neural mechanisms of learning: an analysis of imprinting in the domestic chick. Proc R Soc Lond [Biol] 1981;213:101-37.

3 Salzen EA. Imprinting in birds and primates. Behaviour 1967:28:232-54

Salk L. Thoughts on the concept of imprinting and its place in early humen development. Canadian Psychiatric Association fournal 1966;11:295-305.

deve Peobson B, Eklund G, Hamberger L, Linnarsson D, Sedvall G, Valverius M Perinatal origin of adult self-destructive behavior. Acta Psychiatr Scand
1987;76:364-71.

6 Jacobson B, Nyberg K, Eklund G, Bygdeman M, Rydberg U. Obstetric pain medication and eventual adult amphetamine addiction in offspring. Acta Obstet (ivnnecol Scand 1988;67:677-82.

7 Breslow NE, Day NE. Statistical methods in cancer research. Lyons: World Health Organisation, 1980:248-79. (International Agency for Research on Cancer Scientific Publication No 32, vol 1.)

8 Belfrage P, Boréus LO, Hartvig P, Irestedt L, Raabe N. Neonatal depression after obstetrical analgesia with pethidine. Acta Obstet Gynecol Scand $1981 ; 60: 43-9$

9 Hynes MD, Berkowitz BA. Catecholamine mechanisms in the stimulation of mouse locomotor activity by nitrous oxide and morphine. Eur 7 Pharmacol 1983;90:109-14.

10 Jönsson LE, Gunne LM, Änggard E. Effects of alphamethyltyrosine in amphetamine-dependent subjects. Pharmacologica Clinica 1969:2:27-9.

11 Lagercrantz H, Slotkin TA. The "stress" of being born. Sci Am 1986;254: $92-102$

12 Kovach JK. Effects of autonomic drugs on imprinting. Iournal of Comparative and Physiological Psychology 1964;57:183-7.

13 Brazelton TB. Effect of perinatal drugs on the behavior of the neonate. Am f Psychiatry 1970;126:1261-6.

14 James H. Imprinting with visual flicker: effects of testosterone cyclopentylpropionate. Animal Behaviour 1962;10:341-6.

(Accepted 31 August 1990)

\title{
Can prepregnancy care of diabetic women reduce the risk of abnormal babies?
}

\author{
Judith M Steel, Frank D Johnstone, David A Hepburn, Alistair F Smith
}

Royal Infirmary of Edinburgh, Edinburgh EH3 9YW

Judith M Steel, FRCPED, associate specialist, diabetic department

David A Hepburn, MRCP, registrar, diabetic department Alistair F Smith, FRCPATH senior lecturer, department of clinical chemistry

Department of Obstetrics, University of Edinburgh,

Edinburgh

Frank D Johnstone, FRCOG, senior lecturer

Correspondence to:

Dr Steel.

Br Med f 1990;301:1070-4

\section{Abstract}

Objective - To see whether a prepregnancy clinic for diabetic women can achieve tight glycaemic control in early pregnancy and so reduce the high incidence of major congenital malformation that occurs in the infants of these women.

Design-An analysis of diabetic control in early pregnancy including a record of severe hypoglycaemic episodes in relation to the occurrence of major congenital malformation among the infants.

Setting-A diabetic clinic and a combined diabetic and antenatal clinic of a teaching hospital.

Patients-143 Insulin dependent women attending a prepregnancy clinic and 96 insulin dependent women managed over the same period who had not received specific prepregnancy care.

Main outcome measure-The incidence of major congenital malformation.

Results-Compared with the women who were not given specific prepregnancy care the group who attended the prepregnancy clinic had a lower haemoglobin $A_{I}$ concentration in the first trimester $(8.4 \% v$ $10.5 \%)$, a higher incidence of hypoglycaemia in early pregnancy (38/143 women $v 8 / 96$ ), and fewer infants with congenital abnormalities $(2 / 143 v 10 / 96$; relative risk among women not given specific prepregnancy care $7 \cdot 4(95 \%$ confidence interval 1.7 to $33 \cdot 2)$ ).

Conclusion-Tight control of the maternal blood glucose concentration in the early weeks of pregnancy can be achieved by the prepregnancy clinic approach and is associated with a highly significant reduction in the risk of serious congenital abnormalities in the offspring. Hypoglycaemic episodes do not seem to lead to fetal malformation even when they occur during the period of organogenesis.

\section{Introduction}

There is a high incidence of congenital malformation in infants of diabetic mothers. ${ }^{1.3}$ Both in rats ${ }^{45}$ and in humans ${ }^{6-9}$ the higher are the maternal blood glucose concentrations over the period of organogenesis the greater is the chance of an abnormality in the offspring. In 1976 we started a prepregnancy clinic for diabetic women. The aims of the clinic are manifold, ${ }^{10-12}$ but the most important has become the optimisation of diabetic control over the period of conception in an attempt to reduce the incidence of major congenital abnormalities. Tightening control in early pregnancy inevitably increases the frequency of hypoglycaemia. Recent work with rats has shown that hypoglycaemia at a critical stage of development - namely, equivalent to days 32-40 in humans (18-26 days after ovulation)can cause congenital abnormalities. ${ }^{13-16}$ Hence the desirability of reducing blood glucose concentrations in early pregnancy has been questioned. ${ }^{16}$

This paper reports the first 14 years' experience of our prepregnancy clinic, comparing results in women who attended the clinic with those in a group of women who did not.

\section{Patients and methods}

The prepregnancy clinic is advertised to insulin dependent diabetic women by personal contact, posters, and articles in newsletters. Contraception 